\title{
Aivision of the Swiss Chemical Society
}

\section{Analyzing Breath with Chemical Sensors}

\section{Jan van den Broek and Andreas T. Güntner}

*Correspondence: Dr. A. T. Güntner, Particle Technology Laboratory, Department of Mechanical and Process Engineering, ETH Zürich, Sonneggstrasse 3, CH-8092 Zurich, E-mail: andreas.guentner@ptl.mavt.ethz.ch

\section{Keywords: Gas sensors · Medical diagnostics · Non-invasive ·} Volatile compounds

Non-invasive breath analyzers could facilitate rapid and routine disease screening for early stage detection and improved therapies. In fact, elevated breath concentrations of key molecules have been associated to physiological and pathological states, such as ammonia to kidney failure, acetone to diabetes and enhanced fat metabolism or NO to asthma, the latter being applied actively in today's clinical practice. Especially promising to detect these 'breath markers' are solid-state gas sensors due to their compact design and low cost, making them ideal for incorporation into wearable devices.

In specific, gas sensors based on chemo-resistive metaloxides nanoparticles offer sufficiently low detection limits in the part-per-billion (ppb) range, fast response and recovery times (seconds to few minutes), however, they lack selectivity. This can be tackled for some tracers by material design (e.g. Si-doped $\mathrm{WO}_{3}$ for acetone, Si-doped $\mathrm{MoO}_{3}$ for ammonia), microporous filter membranes or the combination of sensors to arrays.

Recently, a filter-sensor system was developed for fast and highly selective breath isoprene detection. Isoprene is a promising marker for high blood cholesterol levels. The sensor system consists of a filter of activated alumina in combination

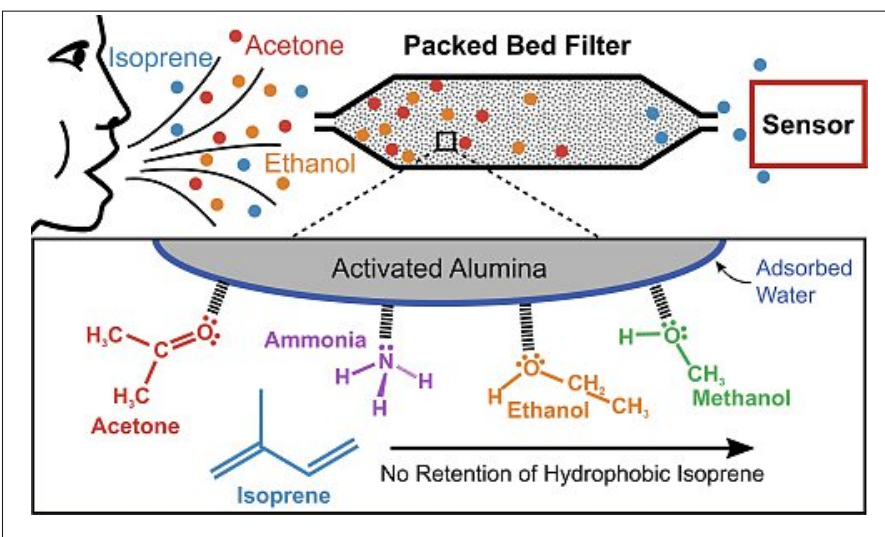

Isoprene detector based on an alumina-powder filter to retain hydrophilic compounds and a highly sensitive Pt-doped $\mathrm{SnO}_{2}$ sensor to quantify isoprene concentrations. Adapted with permission from van den Broek et al., ACS Sens. 2018, 3, 677. Copyright (2018) American Chemical Society. with a non-specific but highly sensitive Pt-doped $\mathrm{SnO}_{2}$ sensor. Isoprene is hydrophobic, in contrast to other major breath compounds including acetone, ammonia, ethanol and methanol. The filter exploits this by ab-/adsorbing and retaining them while isoprene passes unhindered and is registered by the sensor without interference. That way, isoprene is detected quickly $(<5 \mathrm{~s})$ down to $5 \mathrm{ppb}$ with selectivities $>100$ over other compounds in simulated breath mixtures, unprecedented by state-of-the-art sensors. As a result, this sensor-filter system is promising as breath isoprene detector for non-invasive monitoring of high blood cholesterol levels.

Such sensors can be integrated readily into portable breath analyzers for individualized health monitoring at home. This was demonstrated recently with breath acetone sensors that monitored individual fat burn rates in $\mathbf{2 0}$ volunteers during exercise and rest.

\section{Acknowledgement}

The authors thank Prof. S. E. Pratsinis (ETH Zurich) for his support and guidance during this research. Financial support by the SNF Grant \#159763, 170729, and 175754 is kindly acknowledged.

\section{References}

J. van den Broek, A. T. Güntner, S. E. Pratsinis, ACS Sens. 2018, 3, 677.

A. T. Güntner, S. Abegg, K. Wegner, S. E. Pratsinis, Sens. Actuators B 2018 , 257, 916.

A. T. Güntner, N. A. Sievi, S. J. Theodore, T. Gulich, M. Kohler, S. E. Pratsinis, Anal Chem. 2017, 89, 10578.

A. T. Güntner, N. J. Pineau, P. Mochalski, H. Wiesenhofer, A. Agapiou, C A. Mayhew, S. E. Pratsinis, Anal. Chem. 2018, 90, 4940, doi: 10.1021/acs. analchem.8b00237.

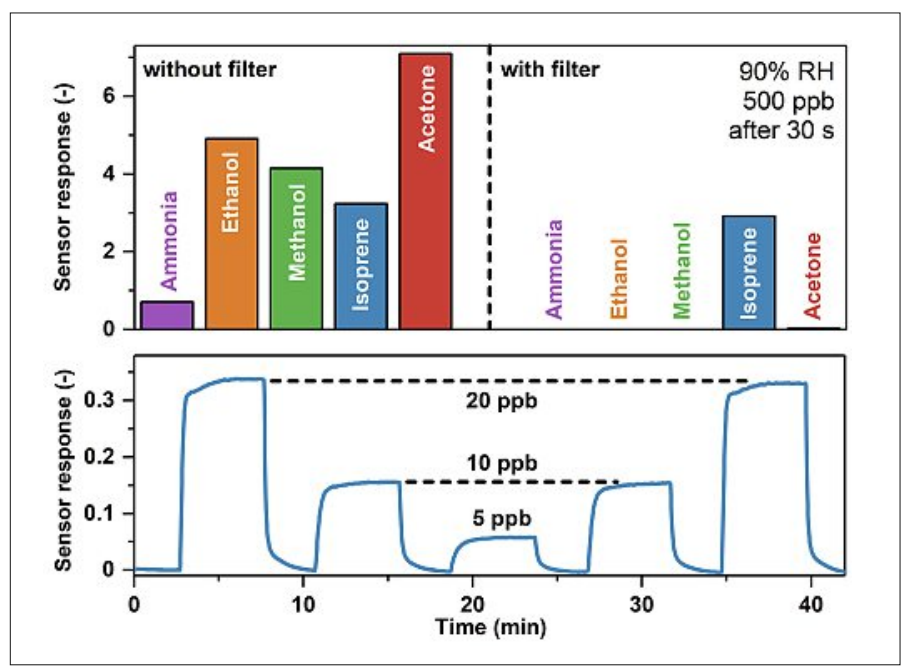

Response of the Pt-doped $\mathrm{SnO}_{2}$, sensor without (top left) and with activated alumina filter (top right) to $500 \mathrm{ppb}$ of breath-relevant analytes at $90 \% \mathrm{RH}$. Response of the filter-sensor system to ultra-low isoprene concentrations of 5, 10 and $20 \mathrm{ppb}$ at $90 \% \mathrm{RH}$ (bottom). Adapted with permission from van den Broek et al., ACS Sens. 2018, 3, 677. Copyright (2018) American Chemical Society. 